\title{
A Novel, Free-Space Optical Interconnect Employing Vertical-Cavity Surface Emitting Laser Diodes and InGaAs Metal-Semiconductor-Metal Photodetectors for Gbit/s RF/Microwave Systems
}

Gregory R. Savich

University of Rochester, Rochester, New York

Rainee N. Simons

Glenn Research Center, Cleveland, Ohio 


\section{NASA STI Program . . . in Profile}

Since its founding, NASA has been dedicated to the advancement of aeronautics and space science. The NASA Scientific and Technical Information (STI) program plays a key part in helping NASA maintain this important role.

The NASA STI Program operates under the auspices of the Agency Chief Information Officer. It collects, organizes, provides for archiving, and disseminates NASA's STI. The NASA STI program provides access to the NASA Aeronautics and Space Database and its public interface, the NASA Technical Reports Server, thus providing one of the largest collections of aeronautical and space science STI in the world. Results are published in both non-NASA channels and by NASA in the NASA STI Report Series, which includes the following report types:

- TECHNICAL PUBLICATION. Reports of completed research or a major significant phase of research that present the results of NASA programs and include extensive data or theoretical analysis. Includes compilations of significant scientific and technical data and information deemed to be of continuing reference value. NASA counterpart of peer-reviewed formal professional papers but has less stringent limitations on manuscript length and extent of graphic presentations.

- TECHNICAL MEMORANDUM. Scientific and technical findings that are preliminary or of specialized interest, e.g., quick release reports, working papers, and bibliographies that contain minimal annotation. Does not contain extensive analysis.

- CONTRACTOR REPORT. Scientific and technical findings by NASA-sponsored contractors and grantees.
- CONFERENCE PUBLICATION. Collected papers from scientific and technical conferences, symposia, seminars, or other meetings sponsored or cosponsored by NASA.

- SPECIAL PUBLICATION. Scientific, technical, or historical information from NASA programs, projects, and missions, often concerned with subjects having substantial public interest.

- TECHNICAL TRANSLATION. Englishlanguage translations of foreign scientific and technical material pertinent to NASA's mission.

Specialized services also include creating custom thesauri, building customized databases, organizing and publishing research results.

For more information about the NASA STI program, see the following:

- Access the NASA STI program home page at http://www.sti.nasa.gov

- E-mail your question via the Internet to help@sti.nasa.gov

- Fax your question to the NASA STI Help Desk at 301-621-0134

- Telephone the NASA STI Help Desk at 301-621-0390

- Write to:

NASA STI Help Desk

NASA Center for AeroSpace Information 7121 Standard Drive Hanover, MD 21076-1320 


\section{A Novel, Free-Space Optical Interconnect Employing Vertical-Cavity Surface Emitting Laser Diodes and InGaAs Metal-Semiconductor-Metal Photodetectors for Gbit/s RF/Microwave Systems}

Gregory R. Savich

University of Rochester, Rochester, New York

Rainee N. Simons

Glenn Research Center, Cleveland, Ohio

Prepared for

Photonics West 2006

sponsored by the International Society for Optical Engineering San Jose, California, January 21-26, 2006

National Aeronautics and

Space Administration

Glenn Research Center

Cleveland, Ohio 44135 


\section{Acknowledgments}

The authors wish to acknowledge Heidi Torres, Polytechnic University of Puerto Rico, for her contributions to the design and theoretical response of the MSM photodetectors as a NASA Glenn LERCIP intern during the summer of 2003. The authors also wish to acknowledge Elizabeth McQuaid and Nicholas Varaljay for their help and extensive work in the clean room, and

Bruce Viergutz for his technical work as well. The authors also wish to express appreciation for the ellipsometric measurements conducted by S. Alterovitz on the photodetector wafers. The NASA Glenn LERCIP internship program supported Heidi Torres during the summer of 2003 and Gregory Savich during the summer of 2004 and 2005 . The NASA

Glenn Research Center Independent Research and Development (IR\&D) Program supported this task (IRD04-32).

This report contains preliminary findings, subject to revision as analysis proceeds.

Level of Review: This material has been technically reviewed by technical management.

Available from

NASA Center for Aerospace Information 7121 Standard Drive

Hanover, MD 21076-1320
National Technical Information Service 5285 Port Royal Road Springfield, VA 22161 


\title{
A Novel, Free-Space Optical Interconnect Employing Vertical-Cavity Surface Emitting Laser Diodes and InGaAs Metal-Semiconductor-Metal Photodetectors for Gbit/s RF/Microwave Systems
}

\author{
Gregory R. Savich \\ University of Rochester \\ Rochester, New York 14627 \\ Rainee N. Simons \\ National Aeronautics and Space Administration \\ Glenn Research Center \\ Cleveland, Ohio 44135
}

\begin{abstract}
Emerging technologies and continuing progress in research are making way for novel, high speed forms of optical data transfer. Vertical-cavity surface emitting laser (VCSEL) diodes operating at $1550 \mathrm{~nm}$ have only recently become commercially available, while metal-semiconductor-metal (MSM) photodetectors are pushing the limits of contact lithography with interdigitated electrode widths reaching sub micron levels. We propose a novel, free-space optical interconnect operating up to $1 \mathrm{Gbit} / \mathrm{s}$ utilizing commercially available $1550 \mathrm{~nm}$ VCSEL diodes and newly fabricated InGaAs MSM photodetectors with functionality for both chip level and board level applications. We report on development, progress, and current work. Analyses of the divergent behavior and of the normalized frequency response of VERTILAS GmbH $1550 \mathrm{~nm}$ VCSEL diodes for coupling to MSM photodetectors with a 50 by $50 \mu \mathrm{m}$ active area are presented. The MSM photodetectors are fabricated on a pseudomorphic $\operatorname{In}_{0.9} \mathrm{Ga}_{0.1} \mathrm{P}-\mathrm{InP}$ InGaAs heterostructure and have gold interdigitated Shottky contacts with varying electrode width and spacing on the order of 1 to $3 \mu \mathrm{m}$. We discuss the calculated response of these MSM photodetectors as well as the fabrication and characterization of the devices. A report on bit error rate (BER) characteristics of the VCSEL diodes coupled to commercially available high-speed photodetectors and successively coupled to the novel MSM photodetectors integrated with commercially available transimpedance amplifiers (TIA) follows. The work accounted here will lead to the formation and characterization of a fully integrated $1 \mathrm{Gbit} / \mathrm{s}$ free-space optical interconnect applying VCSEL diodes and MSM photodetectors operating at $1550 \mathrm{~nm}$ for $\mathrm{RF} /$ microwave digital systems.
\end{abstract}

\section{Introduction}

Free-space optical interconnects remain a topic of fervent discussion and significant research, but much work is still required if free-space optical interconnects are to become practical and commercially viable tools for computing and data transfer. Optical interconnects have been praised for both their speed and functionality (table 1) with hopes that light can relieve the electrical bottleneck many in research and industry are predicting for the near future. VCSEL diodes operating in the near-infrared are now commercially attainable but InGaAs MSM photodetectors are still in need of research and further development. High speed, small area MSM photodetectors will allow dense photodetector arrays and provide added practicality to interconnects, without compromising overall performance. 
TABLE 1.-COMPARISON OF ELECTRICAL AND OPTO-ELECTRONIC INTERCONNECTS CHARACTERISTICS

\begin{tabular}{|l|c|c|}
\hline \multicolumn{1}{|c|}{ Parameter } & Electrical interconnect & Optical interconnect \\
\hline Bandwidth (GHz) & 0.25 & $>2.5$ \\
\hline Cross-talk (isolation) dB & 25 to 30 & $>80$ \\
\hline Speed & $1 \mathrm{~ns}$ & $1 \mathrm{ps}$ \\
\hline Power consumption & Few $\mathrm{mW}$ & Few $\mu \mathrm{W}$ \\
\hline Density of interconnects & 10 to $12 / \mathrm{mm}^{2}$ & $>100 / \mathrm{mm}^{2}$ \\
\hline
\end{tabular}

We report on progress in creating a novel, free-space optical interconnect for Gbit/sec RF/microwave systems. Such an interconnect requires operation at high frequency with low RF/microwave losses at both the input and output end of the link while maintaining a large dynamic range (ref. 1). For these reasons, we chose to use VCSEL diodes which are praised for their low power consumption, single mode operation, and relative ease of fabrication while having very large bandwidths (refs. 2 to 4). For detection, MSM photodetectors are the clear choice. MSM photodetectors have low overall capacitance when compared to detectors of the $\mathrm{P}-\mathrm{i}-\mathrm{N}$ type resulting in a large area detector with bandwidths to match that of the VCSEL diodes (ref. 5). We chose to design and fabricate our own MSM photodetectors for this interconnect, but for other components, an emphasis on using commercially available parts was maintained. Such an emphasis keeps cost and development time to a minimum while making adaptation of the free-space interconnect as a commercially viable tool more feasible and inexpensive. We procured VCSEL diodes operating at $1550 \mathrm{~nm}$ fabricated and packaged by VERTILAS GmbH (Model VCL1550) and also a VCSEL diode mount (Model TCLDM9) with temperature/current controller (Model TED200) from Thorlabs, Inc. We present a discussion on MSM photodetector fabrication, theoretical response, and experimental characterization, followed by a report on the characterization of the VERTILAS VCSEL diodes. The paper concludes with a discussion of the work and analyses done to create the optical interconnect as well as current and planned work for the project.

\section{Metal-Semiconductor-Metal Photodetectors}

MSM photodetectors are chosen over P-i-N or avalanche photodetectors for a number of reasons. First and foremost, MSM photodetectors have a comparatively low capacitance per unit area compared to $\mathrm{P}-\mathrm{i}-\mathrm{N}$ structures. Because of this, high speed MSM photodetector operation is possible with a relatively large active area compared to other photodetectors. A larger active area decreases the alignment accuracy required for successful operation, yet MSM photodetectors boast high speed operation from a detector with a larger area than most P-i-N detectors (ref. 5). The reported high speed capabilities of MSM detectors provide another significant advantage over P-i-N detectors. The trade-off in this case comes from the shadowing effects caused by the fingers of an MSM photodetector. Shadowing effects have been shown to greatly reduce the responsivity of the detectors. To overcome this effect, we have chosen to fabricate MSM photodetectors with semi-transparent, gold ( $\mathrm{Au}$ ), interdigitated Schottky contacts, a strategy first presented by Rong-Heng Yuang, et al. (ref. 6). We chose to fabricate a series of square detectors, $50 \mu \mathrm{m}$ on each side, with varying electrode finger widths and finger spacings for characterization and implementation into a free-space optical interconnect. Circular MSM photodetectors have been shown to have a lower overall capacitance and an improved frequency response (ref. 7) and, therefore, may become a topic of further study pending the outcome of the square MSM characterization. We report on fabrication, theoretical response, and current experimental progress of the MSM photodetectors.

\subsection{MSM Photodetector Fabrication}

The MSM photodetectors are fabricated on a 2 in., Fe doped-InP wafer layered with a pseudomorphic $\mathrm{In}_{0.9} \mathrm{Ga}_{0.1} \mathrm{P}$-InP-InGaAs heterostructure. EpiWorks, Inc., at their commercial facility deposited to our specifications the epitaxial layers on the InP substrate. Upon receiving the wafers, ellipsometry was used 
to confirm the integrity of the wafer and verify our specifications were met. The ellipsometric measurements showed that the epitaxial structure of the wafers were near ideal. A $0.5 \mu \mathrm{m}$, undoped layer of $\mathrm{InP}$ was deposited on the InP substrate to serve as a buffer for the active layer. The $1 \mu \mathrm{m}$ active layer is comprised of undoped InGaAs, which provides excellent responsivity at the target wavelength of $1550 \mathrm{~nm}$. Two barrier enhancement layers, an undoped InP layer $40 \mathrm{~nm}$ thick and an undoped $\operatorname{In}_{0.9} \operatorname{Ga}_{0.1} \mathrm{P}$ that is $10 \mathrm{~nm}$ thick cover the InGaAs layer. The semi-transparent, gold interdigitated Schottky contacts are deposited on this construction. Further information on the epitaxial structure can be found in RongHeng Yuang, et al. (ref. 6).

The Au electrodes sit on top of a mesa that extends through all five upper layers down to the substrate providing electrical isolation for the photodetector. An antireflective coating of silicon nitride is deposited on top of the detector mesa, and a 1.5- $\mu$ m-thick coplanar waveguide (CPW) serves as an electrical connection to the Schottky contacts (fig. 1) (ref. 8). The MSM photodetectors have an active area of 50 by $50 \mu \mathrm{m}$ with Au fingers varying from 1 to $3 \mu \mathrm{m}$ set apart by spacing also varying from 1 to $3 \mu \mathrm{m}$ totaling 9 different designs. The design of the detector without the CPW covers a space approximately 100 by $50 \mu \mathrm{m}$ taking into account the Au pads that connect the detector to the CPW (fig. 2).

To fabricate the detectors, the $10 \mathrm{~nm}$ thick Au electrodes were first deposited on the wafer after mask alignment and photoresist exposure using contact microlithography. Then after aligning the mesa mask, three separate chemical etches were performed to create the isolation mesa for the device. The first etch was a fast etch of $\mathrm{HCl}: \mathrm{H}_{2} \mathrm{O}=7: 1$ concentration that removed the $\mathrm{In}_{0.9} \mathrm{Ga}_{0.1} \mathrm{P}$ layer and upper InP layer. Second, a slow etch composed of $\mathrm{H}_{3} \mathrm{PO}_{4}: \mathrm{H}_{2} \mathrm{O}_{2}: \mathrm{H}_{2} \mathrm{O}=4: 3: 1$ concentration was used to remove the InGaAs active later. At this point, the mesa photoresist was removed and then replaced to ensure protection of the $\mathrm{Au}$ electrodes. Finally, another $\mathrm{HCl}: \mathrm{H}_{2} \mathrm{O}=7: 1$ etch was used to remove the undoped InP, achieve full electric isolation, and reach the substrate material. The silicon nitride was then deposited to a thickness of $220 \mathrm{~nm}$. Finally, the $1.5 \mu \mathrm{m}$ gold CPW was deposited to complete the fabrication process. Figure 3 shows a close-up of the active area of a detector with $1 \mu \mathrm{m}$ fingers and $1 \mu \mathrm{m}$ finger spaces. The white area on either side of the active region is the CPW and the slightly darker region over the Au fingers is the silicon nitride anti-reflective coating. Notice the semi-transparency of the fingers. Figure 4 provides a view of the entire mesa surrounded by the CPW. The dark center area is the active region with the fingers covered in the silicon nitride coating.

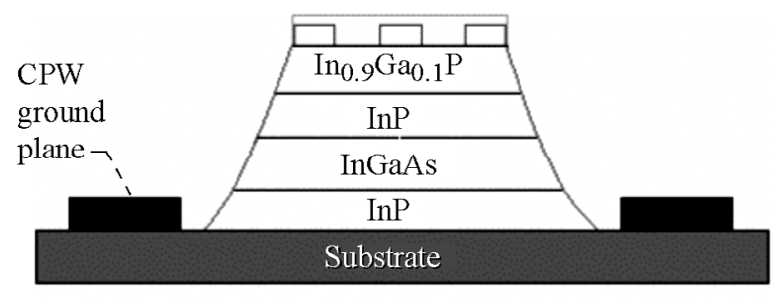

Figure 1.- Vertical mesa structure of the MSM photodetector with CPW ground plane on either side.

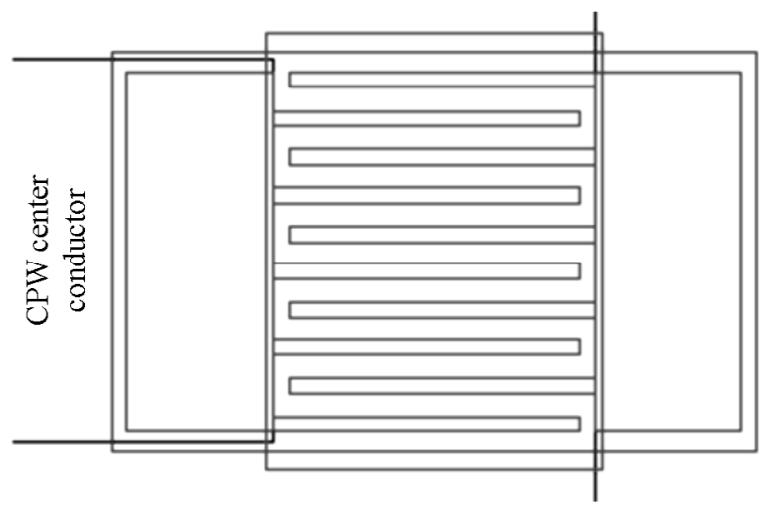

Figure 2.-Layout of MSM photodetector showing the area of the mesa, and the contact with the CPW center conductor. 


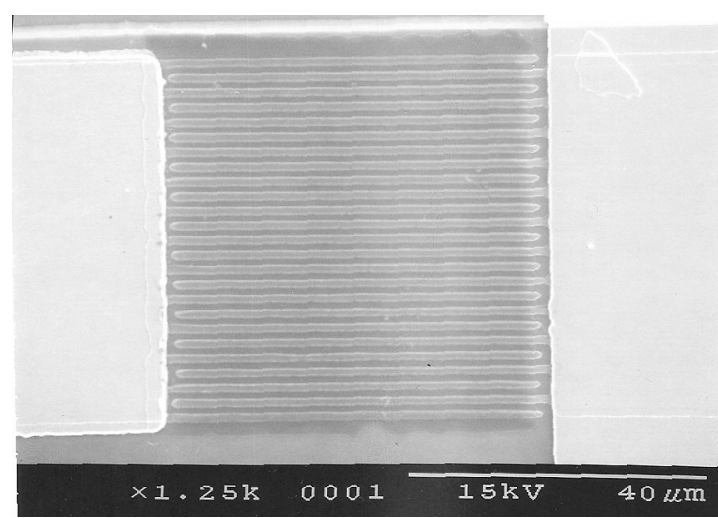

Figure 3.-Close-up view of the active area of the MSM photodetector with $1 \mu \mathrm{m}$ finger width and $1 \mu \mathrm{m}$ finger spacing.

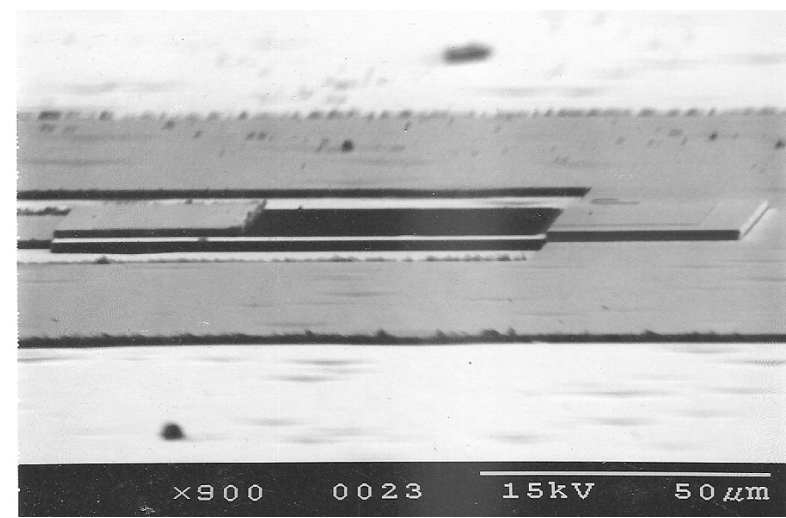

Figure 4.- -View of fabricated MSM photodetector showing the detector on the mesa surrounded by the CPW.

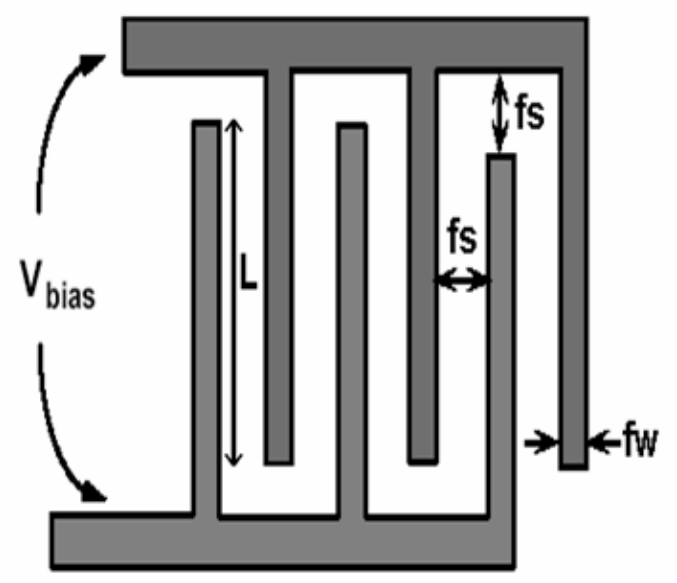

Figure 5.-MSM photodetector concept with design variables defined.

\subsection{Theoretical Calculated Response}

The theoretical response of the MSM photodetectors was calculated. We determined the expected total capacitance, transit time, bandwidth, external quantum efficiency, and responsivity of the detectors given their respective finger width and finger spacing. Both the finger width and finger spacing vary in integer values from 1 to $3 \mu \mathrm{m}$, with all possible permutations being studied. The size of the active area remains a square, $50 \mu \mathrm{m}$ on each side, regardless of the photodetector spacing characteristics. The finger width, finger spacing, and active area length are given by $f_{w}, f_{s}$, and $L$, respectively (fig. 5).

The total capacitance, $C$, of the MSM photodetectors is given (ref. 9) by,

$$
C=\frac{K(k)}{K\left(k^{\prime}\right)} \cdot \varepsilon_{o} \cdot\left(1+\varepsilon_{r}\right) \cdot \frac{A}{f_{s}+f_{w}} \quad \text { Farads }
$$

Where $\varepsilon_{o}$ is the permittivity of free space equal to $8.8542 \times 10^{-12} \mathrm{~F} / \mathrm{m}$ and $\varepsilon_{r}$ is the relative permittivity. The quantity $A$ is defined as the active area of the detector given, in this case, by the expression $A=L^{2}$. In order to solve this equation, the ratio $K(k) / K\left(k^{\prime}\right)$ must be determined. $K(k)$ is defined as the complete elliptical integral of the first kind and is given by the expression, 


$$
K(k)=\int_{0}^{\pi / 2} \frac{1}{\sqrt{1-k^{2} \sin ^{2} \phi}} d \phi
$$

We define $k$ and $k^{\prime}$ as,

$$
k=\tan ^{2}\left[\frac{\pi \cdot f_{w}}{4 \cdot\left(f_{S}+f_{w}\right)}\right]
$$

and,

$$
k^{\prime}=\sqrt{1-k^{2}}
$$

respectively (ref. 9). Using these definitions, we determine for a given photodetector geometry the $k$ and $k^{\prime}$ values. The ratio $K(k) / K\left(k^{\prime}\right)$ is given by the expression

$$
\frac{K(k)}{K\left(k^{\prime}\right)}=\frac{1}{2 \pi} \cdot \ln \left[2 \cdot\left(\frac{\sqrt{1+k}+\sqrt[4]{4 \cdot k}}{\sqrt{1+k}-\sqrt[4]{4 \cdot k}}\right)\right]
$$

for the case where $K(k) / K\left(k^{\prime}\right)$ is between one and infinity and $k$ is between $1 / \sqrt{ }(2)$ and one (ref. 10). The transit time, $t_{T}$ for the MSM photodetectors is given by the expression,

$$
t_{T}=\frac{f_{s}}{2 \cdot V_{s a t}} \quad \text { seconds }
$$

Where $V_{\text {sat }}$ is defined as the carrier saturation velocity. The bandwidth, $B W$, of the device is found using

$$
B W=\frac{14.17}{f_{s}}+0.314 \quad \mathrm{GHz}
$$

and the external quantum efficiency, $\eta$, can be arrived at through the equation

$$
\eta=(1-r) \cdot\left(1-e^{\frac{-d}{\delta_{O P T}}}\right) \cdot \frac{f_{S}}{f_{S}+f_{w}}
$$

Where $r$ is the Fresnel reflectivity, $d$ is the absorption layer thickness and $\delta_{O P T}$ is defined as the optical penetration depth (ref. 11). Finally, the responsivity, $R$, is given by

$$
R=\eta q \cdot \frac{\lambda}{h c} \quad \mathrm{~A} / \mathrm{W}
$$

Where $\eta$ is the previously calculated external quantum efficiency, $q$ is the charge of an electron, $\lambda$ is the wavelength of the photons to be detected, $h$ is Planck's constant, and $c$ is the speed of light in a vacuum. The results of the analysis are presented in table 2 . 
TABLE 2.-THEORETICAL RESPONSE OF THE MSM PHOTODETECTORS

FOR VARIOUS FINGER WIDTHS AND SPACING

\begin{tabular}{|c|c|c|c|c|c|c|c|c|c|}
\hline $\mathbf{f}_{\mathbf{w}} \times \mathbf{f}_{\mathbf{s}}(\boldsymbol{\mu m})$ & $\mathbf{1} \times \mathbf{1}$ & $\mathbf{1} \times \mathbf{2}$ & $\mathbf{1} \times \mathbf{3}$ & $\mathbf{2} \times \mathbf{1}$ & $\mathbf{2} \times \mathbf{2}$ & $\mathbf{2} \times \mathbf{3}$ & $\mathbf{3} \times \mathbf{1}$ & $\mathbf{3} \times \mathbf{2}$ & $\mathbf{3} \times \mathbf{3}$ \\
\hline $\mathbf{C}(\mathbf{f F})$ & 71.90 & 37.50 & 24.50 & 61.30 & 35.90 & 24.90 & 52.80 & 33.30 & 23.90 \\
\hline $\mathbf{t}_{\mathbf{T}} \mathbf{( p s )}$ & 8.33 & 16.70 & 25.00 & 8.33 & 16.70 & 25.00 & 8.33 & 16.70 & 25.00 \\
\hline $\mathbf{B W}(\mathbf{G H z})$ & 14.50 & 7.40 & 5.04 & 14.50 & 7.40 & 5.04 & 14.50 & 7.40 & 5.04 \\
\hline $\boldsymbol{\eta}$ & 0.22 & 0.29 & 0.33 & 0.15 & 0.22 & 0.27 & 0.11 & 0.18 & 0.02 \\
\hline $\mathbf{R}(\mathbf{A} / \mathbf{W})$ & 0.28 & 0.37 & 0.42 & 0.19 & 0.28 & 0.33 & 0.14 & 0.22 & 0.28 \\
\hline
\end{tabular}

Our analysis shows that as the finger spacing increases, the capacitance decreases as would be expected. On the other hand, an increase in $f_{s}$ also results in a decrease in bandwidth and increase in transit time. The results suggest that the most responsive detector will have a finger width of $1 \mu \mathrm{m}$ with a finger spacing of $3 \mu \mathrm{m}$, but this detector will also suffer from the smallest bandwidth. On the contrary, the detector with $3 \mu \mathrm{m}$ finger widths and $1 \mu \mathrm{m}$ finger spaces should have the smallest responsivity but the largest bandwidth. Finding the ideal MSM photodetector will obviously involve a tradeoff justified by the application of the device.

\subsection{Experimental Response and Characterization}

Experimental testing of the fabricated photodetectors has recently begun and is currently a work in progress. A small number of photodetectors on a small piece of InP wafer were fabricated to the point prior to silicon nitride deposition. The wafer piece presented photodetectors complete except for the antireflective coating and CPW. One of this small set of detectors survived wire bonding and was mounted for curve tracing. The curve tracer was used to find the dark I-V characteristic of the detector with $1 \mu \mathrm{m}$ finger width and $2 \mu \mathrm{m}$ finger spacing (fig. 6). The dark I-V characteristic is defined as the characteristic of the detector without light incident on the active area at time of testing.

The I-V curve shows the steep, asymptotic behavior one would expect from a diode of this type in the forward bias direction. The reverse bias side shows a relatively slower, but still significant increase over voltage. The reverse bias increase does not exactly match the exponential behavior of the forward bias region, as it increases at a significantly slower rate, but the diode response in both the forward and reverse bias directions is typical of MSM photodetectors with interdigitated Schottky contacts (ref. 12). Note that the curve intersects the origin as is expected in a dark I-V characteristic. It is expected that with the application of a laser beam at $1550 \mathrm{~nm}$, the curve will begin to move into the negative current quadrants. Further experiments will be conducted to verify this hypothesis.

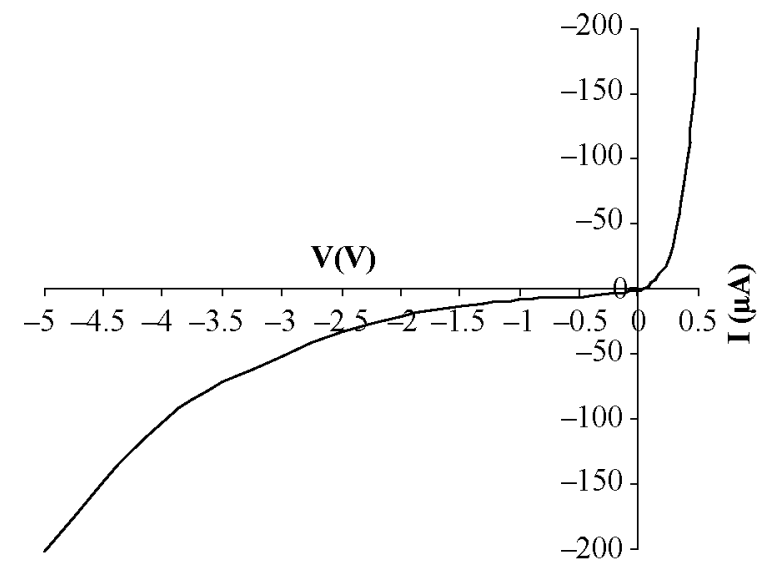

Figure 6.-Measured I-V characteristic of an MSM photodetector with $1 \mu \mathrm{m}$ finger widths and $2 \mu \mathrm{m}$ finger spacing. 


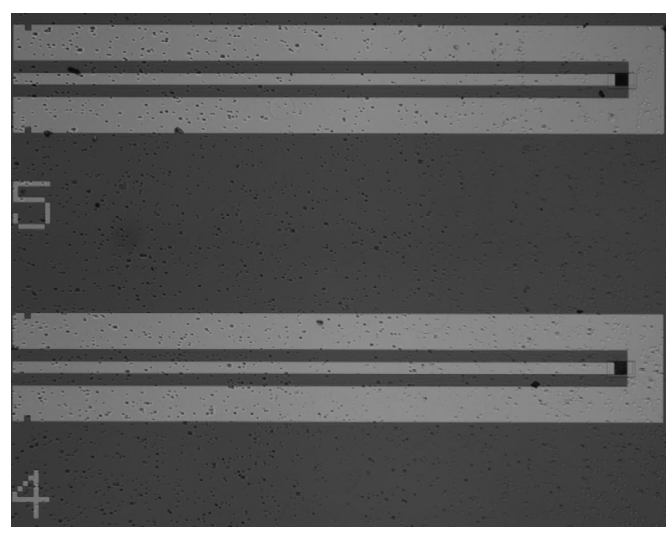

Figure 7.-Two photodetectors with their respective CPWs fully fabricated on the wafer.

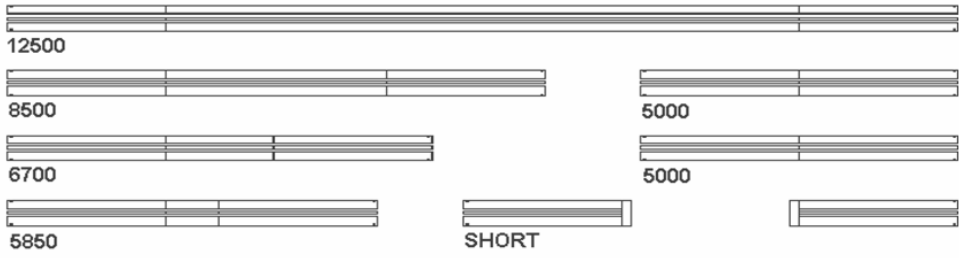

Figure 8.-The design for the set of calibration standards integrated directly onto the wafer, adjacent to the set of photodetectors.

Concurrently, experiments will be conducted to determine the RF response of the MSM photodetectors. For this reason, the photodetectors have been fabricated with an integrated CPW on each detector (fig. 7). In figure 7, the photodetectors are the small black squares on the right side of the CPW. The small indentations on the left side of the CPW are alignment markers for a probe tip. In order to ensure accurate results, on wafer calibration standards have been added to the set of photodetectors as well (fig. 8).

The results of the RF analysis and characterization will be quite useful for determining the level of performance that can be expected from the MSM photodetectors and the optical interconnects into which they will be integrated.

\section{Vertical-Cavity Surface Emitting Laser Diodes}

For free-space optical interconnects, VCSEL diodes provide significant advantages over traditional edge-emitting laser diodes. Edge-emitting diodes must be cut and mounted after being fabricated, while VCSEL diodes can lase directly on the wafer. It is also possible to create arrays of VCSEL diodes directly on the wafer, a virtual impossibility for the edge-emitting types. It is significantly more difficult to fabricate arrays of edge-emitting diodes as each diode must be mounted individually, decreasing the possible density of the array. Furthermore, VCSEL diodes can be fabricated on the same wafer as the MSM photodetectors, increasing the functionality of an array of interconnects. It is not possible to fabricate edge-emitting diodes with MSM detectors into such an array on a single wafer. Furthermore, VCSEL diodes have been praised for their single mode operation, low threshold currents, relatively low power consumption, high coupling efficiency, and the possibility of low cost manufacturing (refs. 2 and 4). The possibilities for increased VCSEL functionality are just now being explored. Integrating microelectro-mechanical systems (MEMS) devices in the form of a movable mirror membrane onto a VCSEL diode to create a laser with a tuning range of over $40 \mathrm{~nm}$ was recently reported and the integration of MEMS controlled micro-lenses may provide an effective means of on wafer beam steering for arrays of free-space optical interconnects (ref. 13).

Only recently have VCSEL diodes operating at $1550 \mathrm{~nm}$ become available commercially, packaged and ready for integration. Rather than develop and fabricate a new VCSEL diode, we chose to use a commercially available product for creating the optical interconnect. This choice provided significant advantages over fabricating a new laser. These advantages include saving time and funds, ensuring a reliable and functional laser with known characteristics, and increasing the feasibility and ease of adapting the interconnect for commercial use on the market. We obtained VCSEL diodes operating at $1550 \mathrm{~nm}$ from VERTILAS GmbH. These diodes laser at a threshold current of about $1 \mathrm{~mA}$, with a 


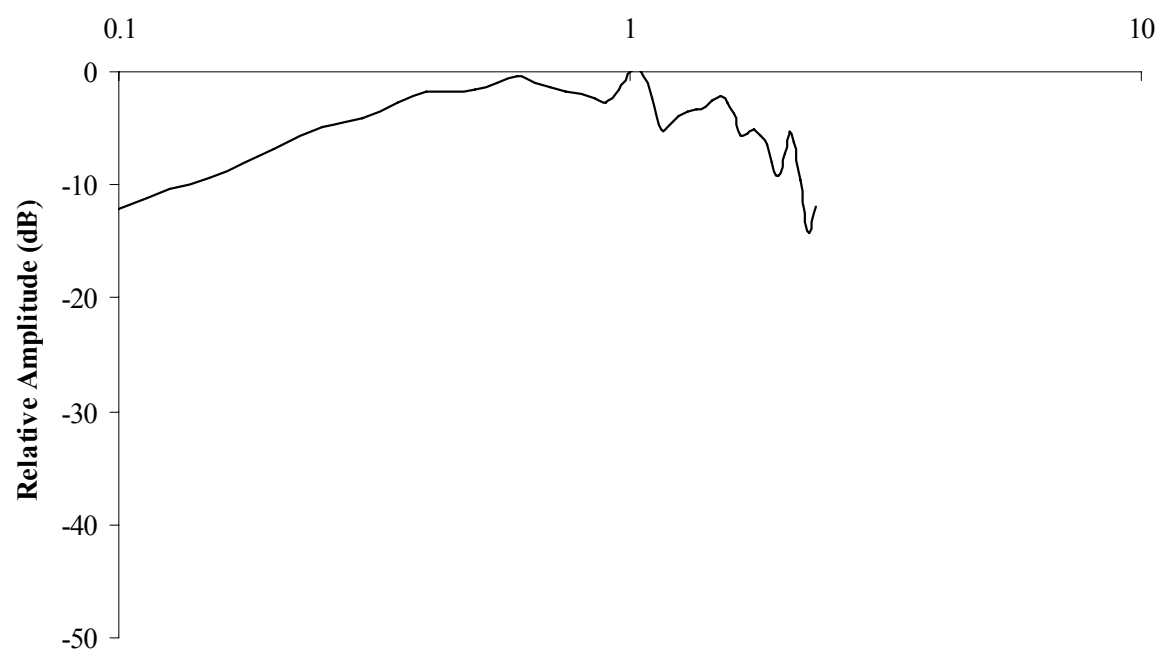

Figure 9.-Measured normalized frequency response of the VCSEL diode mounted in a TEC temperature controlled laser diode mount.

maximum optical power output of about $1 \mathrm{~mW}$ in continuous wave, single mode operation from $-20{ }^{\circ} \mathrm{C}$ to $70{ }^{\circ} \mathrm{C}$. We report on RF characterization and an analysis of the divergence of the VCSEL diode followed by efforts to couple the laser into optical interconnects.

\subsection{Normalized Frequency Response}

We determined the normalized frequency response of the VCSEL diodes to the limits of our equipment. The VCSEL diode was mounted on a thermoelectric cooled (TEC) temperature controlled laser diode mount from Thorlabs, Inc. The laser diode mount is rated up to a modulation rate of $500 \mathrm{MHz}$. The laser is coupled to a single-mode fiber through free space, and the fiber terminates to a high speed photodetector, also from Thorlabs, Inc., that is rated up to a modulation rate of $5 \mathrm{GHz}$. The photodetector output is connected to a spectrum analyzer operational between $50 \mathrm{MHz}$ and $2.3 \mathrm{GHz}$. Figure 9 shows the results of the frequency response experiment.

We expect that further analysis to higher modulation rates will result in the typical $40 \mathrm{~dB} / \mathrm{decade}$ attenuation (ref. 12). An experiment to verify this expected roll-off will be completed upon procurement of a spectrum analyzer with extended range. The integration of a laser mount with increased modulation capabilities would do the most to improve the performance of the current interconnect arrangement. A mount that includes the TEC capabilities and the ease of use of the current mount while operating at higher modulation rates has not been found.

\subsection{Divergence Analysis}

We analyze the divergence of the VCSEL diode beam to determine the ideal coupling distance for a free-space optical interconnect. We assume a Gaussian beam shape with the beam waist at the aperture of the laser. VERTILAS GmbH reports an average aperture radius of $2.5 \mu \mathrm{m}$. The analysis begins with the expression for the radius of a Gaussian beam, $w(z)$, as it travels through space (ref. 14)

$$
w(z)=w_{o} \sqrt{1+\frac{z^{2}}{z_{o}^{2}}}
$$


Where $z$ is the distance along the optical axis, $w_{o}$ is the beam waist at $z=0$, taken to be $2.5 \mu \mathrm{m}$ for our purposes and $z_{o}$ is the Rayleigh range given by

$$
z_{o}=\frac{\pi w_{o}^{2}}{\lambda}
$$

For our purposes, it was desirable to have an expression for the distance along the optical axis as a function of the beam radius rather than the beam radius as a function of the distance along the optical axis. Substituting the expression for the Rayleigh range into equation (10) and then solving for $z$, we obtain

$$
z= \pm \frac{\pi w_{O}}{\lambda} \sqrt{w^{2}(z)-w_{O}^{2}}
$$

Which gives the distance from the aperture of the VCSEL diode for a specific beam radius. In our case, the negative solution is irrelevant as we are only interested in forward beam propagation, so we use

$$
z=\frac{\pi w_{O}}{\lambda} \sqrt{w^{2}(z)-w_{o}^{2}}
$$

to analyze the coupling distances between the VCSEL diodes and the MSM photodetectors in free space.

\section{Optical Interconnects}

The logical conclusion of the characterization analyses of both the VCSEL diodes and the MSM photodetectors is the construction of a free-space optical interconnect for Gbit/sec RF/microwave systems. The first interconnects will be created directly on a probe station from which photodetectors can be switched relatively easily. In this arrangement, it will be possible to test the effectiveness of the various different photodetector designs. We report on coupling progress and analysis as well as planned work.

\subsection{Coupling VCSEL Diodes to a Commercially Available Photodetector}

In order to characterize the procured VCSEL diodes, it became necessary to couple the diode to a commercially available high-speed photodetector. In order to accomplish this, the VCSEL diode was coupled into a $1 \mathrm{~m}$ long, single-mode optical fiber using a series of lenses. The coupling was successful but resulted in a significant loss of optical power at the end of the fiber. Nevertheless, enough power remained to couple the fiber to a high sensitivity, high speed photodetector from Thorlabs, Inc. (Model SIR5-FC) which was then coupled to a spectrum analyzer on which the expected peak was observed for each given modulation rate. It was by this method that the normalized RF frequency response of the VCSEL diode was determined. The signal given by the photodetector may have been sufficient to gain accurate results from the spectrum analyzer, but the signal proved too weak for bit error rate (BER) characterization.

It is desirable to determine the BER characteristics of the VCSEL diode before integrating it with the MSM photodetectors to form an optical interconnect to ensure that the laser can operate with a sufficiently low BER and, therefore, meet the requirements for optical communications. In order to perform this experiment, the coupling efficiency of the link must be improved. Two strategies have been studied to solve the efficiency problem. The first strategy is to improve the efficiency of the coupling between the VCSEL diode and the optical fiber. This can be accomplished by ensuring diffraction limited performance from all lenses in the system and by carefully readjusting the lenses of the system to ensure 
the beam remains on, or close to the optical axis of the coupling apparatus. This ensures that the beam of light remains circular and focuses head on to the fiber tip rather than the beam meeting the fiber at an angle from the optical axis. The second strategy calls for the procurement of a high speed photodetector designed for free space use that can be coupled directly to the laser diode without the use of lenses or fibers. This strategy provides three significant advantages over the first strategy. First, it greatly reduces coupling losses through the link, and second, it is much easier and less time consuming than improving the fiber optic coupling. Third and most importantly, this arrangement approximates the free-space optical interconnect that we intend to create. Such a link would provide a good control for comparison for the free-space optical link with the MSM photodetectors. Despite these advantages, the second strategy presents a fairly significant cost, so efforts to improve the efficiency of the fiber coupling are being examined first. At a point where coupling efficiency has increased enough to allow accurate BER testing, the BER characteristics will be determined.

\subsection{Coupling VCSEL Diodes to MSM Photodetectors}

A coupling plan has been developed to link the VCSEL diodes to the fabricated MSM photodetectors thereby creating the free-space optical interconnect. The main coupling consideration that required attention is the distance between the MSM photodetector and the VCSEL diode, defined as the coupling distance. To determine the ideal coupling distance, the divergence analysis performed on the VERTILAS GmbH VCSEL diodes was used. Two cases were studied, one where the laser beam under-fills the MSM detector and another where the beam over-fills. For the under-filling case, it was assumed that the beam would fill the $50 \mu \mathrm{m}$ width of the detector, but since the beam was circular, would not fill the entire surface of the square detector at full width at half maximum (FWHM). The second case overfilled the detector assuming the FWHM spot size of the laser beam equaled the diagonal of the detector surface equal to $70.71 \mu \mathrm{m}$. At the reported average beam aperture radius of $w_{o}=2.5 \mu \mathrm{m}$, the under-filled coupling distance and the over-filled coupling distance $z$, were found to be 126.04 and $176.70 \mu \mathrm{m}$, respectively. An analysis of the coupling distance was completed for both the 50 and the $70.71 \mu \mathrm{m}$ spot sizes for laser aperture radii up to $5 \mu \mathrm{m}$ (fig. 10). The three aperture radii $2,2.5$, and $3 \mu \mathrm{m}$ were taken to analyze the behavior of the coupling distance as the desired spot size radius, $w_{1}(z)$, is varied up to $50 \mu \mathrm{m}$ (fig. 11).

It was determined that the aperture radius of the VCSEL diode has a dramatic effect on the coupling distance. For the $50 \mu \mathrm{m}$ spot size, the difference between the coupling distances for the 2 and $3 \mu \mathrm{m}$ radius apertures was $49.90 \mu \mathrm{m}$, nearly the difference between under-filling and over-filling the detector. At the $70.71 \mu \mathrm{m}$ spot size, the difference is more dramatic equaling $71.11 \mu \mathrm{m}$. This data will prove useful when the VCSEL diodes are coupled through free-space to the MSM photodetectors. Analyses of both the under-filling and over-filling cases will be studied.

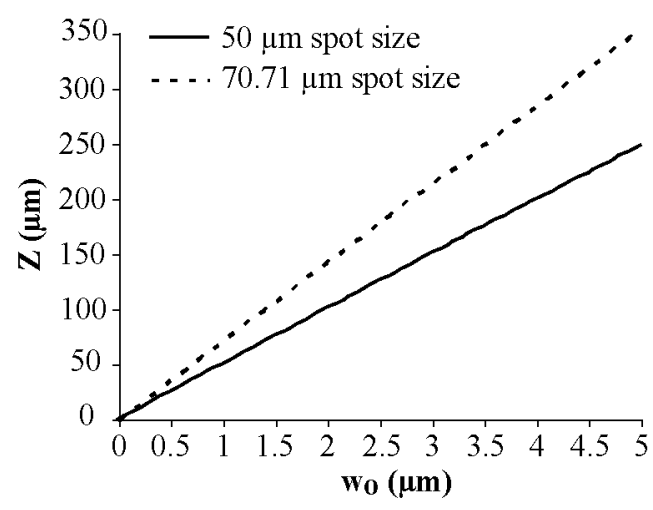

Figure 10.-Coupling distance versus aperture radius for the proposed free-space optical interconnect.

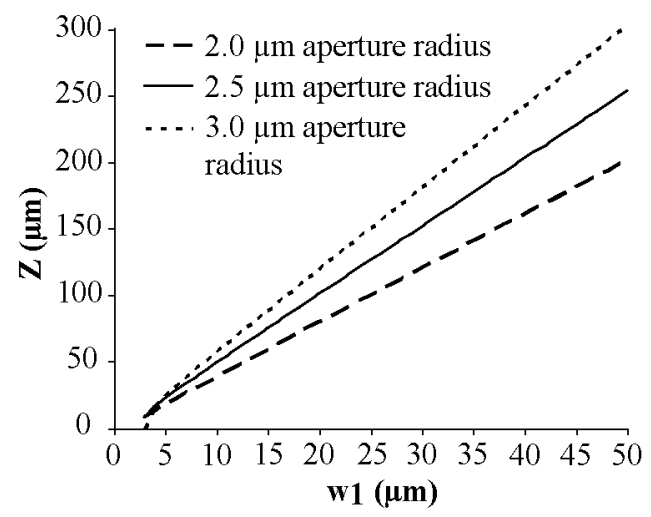

Figure 11.-Coupling distance versus spot radius for the proposed free-space optical interconnect. 


\subsection{Arrays of Optical Interconnects and Beyond}

Once a viable working free-space optical interconnect is demonstrated, work will continue to improve the performance of the link and create a way to package the interconnect in a usable, marketable form. This will involve integrating commercially available transimpedance amplifiers (TIA) into the interconnect to increase functionality for data communications as well as investigating the possibility of integrating MEMS devices, mainly in the form of micro-lenses to steer the laser beam and improve optical coupling efficiency. Further research is planned to study the possibility of creating arrays of interconnects that will integrate MSM photodetectors and VCSEL diodes on the same wafer. Analysis of array densities will be determined as well as the effect of optical and electrical crosstalk. The main source of crosstalk in densely-packed arrays is not the optical coupling between VCSEL but rather the electrical coupling via the conducting substrate. An analysis of the electrical crosstalk in multiple wavelength VCSEL arrays coupled to a single multi-mode fiber has been reported. The results showed that for a densely-packed VCSEL array (with $3 \mu \mathrm{m}$ pitch), the electrical crosstalk between adjacent diodes is less that $-25 \mathrm{~dB}$ up to the $3-\mathrm{dB}$ bandwidth of the diodes (ref. 15). The crosstalk in densely-packed arrays due to electrical coupling can be further reduced by using semi-insulating substrate and also by etching isolating trenches around the diodes. If the VCSEL and MSM photodetector can each be kept isolated in an area 100 by $100 \mu \mathrm{m}$ or smaller, the density of interconnects on a chip could reach 100 interconnects $/ \mathrm{mm}^{2}$ or more. This is far greater than the densities possible with electrical circuits (table 1). These results bode well for dense arrays of interconnects for RF/microwave links. Other research has shown the three major sources of optical crosstalk in a free-space optical interconnect system to be due to diffraction, the reflection of light off of optical surfaces, and the reflection of light off the surface of the photodetectors (ref. 16). These three factors will be intriguing subjects of research for future work on the free-space optical interconnect as will all of the other subjects discussed here.

\section{Conclusions}

We have successfully fabricated 9 different designs of MSM Photodetectors on a 2 in., Fe doped-InP wafer layered with a pseudomorphic $\mathrm{In}_{0.9} \mathrm{Ga}_{0.1} \mathrm{P}-\mathrm{InP}-\mathrm{InGaAs}$ heterostructure for operation at $1550 \mathrm{~nm}$ wavelength. The finger widths and finger spacings of the detectors vary from 1 to $3 \mu \mathrm{m}$ by integer amounts. The theoretical response of the detectors was reported with responsivity ranging from 0.14 to $0.42 \mathrm{~A} / \mathrm{W}$. The total capacitance of the detectors ranges from 23.90 to $71.90 \mathrm{fF}$ and the bandwidth ranges from 5.04 to $14.50 \mathrm{GHz}$. Testing and characterization of the MSM photodetectors has begun and the I-V characteristic of a detector with $1 \mu \mathrm{m}$ finger width and $2 \mu \mathrm{m}$ finger spacing has been reported and shown to be characteristic of MSM photodetectors with Schottky contacts. Progress on characterizing the RF response of a VERTILAS $1550 \mathrm{~nm}$ VCSEL diode was discussed with the normalized frequency response of the device reported. An analysis of the divergence of the laser diodes is complete which is used to determine the coupling distances for the novel free-space optical interconnect. The coupling distance for the average VCSEL aperture radius of $2.5 \mu \mathrm{m}$ is approximately in the range of 126 to $177 \mu \mathrm{m}$. Progress on finding the BER characteristics has found a need to improve the coupling efficiency of the VCSEL diode. This analysis resulted in two viable strategies for laser coupling efficiency improvement including improving the fiber coupling analysis and changing the link to a free-space interconnect. The preceding research in conjunction with the work in progress allows for the creation of a free-space optical interconnect for RF/systems that with continuing efforts will result in a novel Gbit/sec optical data link. 


\section{References}

1. S. Jasmin, N. Vodjdani, J.-C. Renaud, and A. Enard, "Diluted- and Distributed-Absorption Microwave Waveguide Photodiodes for High Efficiency and High Power," IEEE Transactions on Microwave Theory and Techniques, Vol. 45, No. 8, Part 2, pp. 1337-1341, August 1997.

2. M. Ortsiefer, R. Shau, G. Böhm, F. Köhler, and M.-C. Amann, "Low-Threshold Index-Guided $1.5 \mu \mathrm{m}$ Long-Wavelength Vertical-Cavity Surface-Emitting Laser with High Efficiency," Applied Physics Letters, Vol. 76, No. 16, pp. 2179-2181, April 2000.

3. M. Ortsiefer, R. Shau, F. Mederer, R. Michalzik, J. Rosskopf, G. Böhm, F. Köhler, C. Lauer, M. Maute, and M.-C. Amann, "High-Speed Modulation up to $10 \mathrm{Gbit} / \mathrm{s}$ with $1.55 \mu \mathrm{m}$ Wavelength InGaAlAs VCSELs," Electronics Letters, Vol. 38, No. 20, pp. 1180-1181, September 2002.

4. M. Ortsiefer, M. Fürfanger, J. Rosskopf, G. Böhm, F. Köhler, C. Lauer, M. Maute, W. Hoffman, and M.-C. Amann, "Singlemode 1.55 $\mu \mathrm{m}$ VCSELs with Low Threshold and High Output Power," Electronics Letters, Vol. 39, No. 24, pp. 1731-1732, November 2003.

5. S.-W. Seo, S.-Y. Cho, S. Huang, J. J. Shin, N. M. Jokerst, A. S. Brown, and M. A. Brooke, "HighSpeed Large-Area Inverted InGaAs Thin-Film Metal-Semiconductor-Metal Photodetectors," IEEE Journal of Selected Topics in Quantum Electronics, Vol. 10, No. 4, pp. 686-693, July/August 2004.

6. R.-H. Yuang, J.-I. Chyi, Y.-J. Chan, W. Lin, and Y.-K. Tu, "High-Responsivity InGaAs MSM Photodetectors with Semi-Transparent Schottky Contacts," IEEE Photonics Technology Letters, Vol. 7, No. 11, pp.1333-1335, November 1995.

7. R.-H. Yuang, H.-C. Shieh, Y.-J. Chien, Y.-J. Chan, J.-I. Chyi, W. Lin, and Y.-K. Tu, "HighPerformance Large-Area InGaAs MSM Photodetectors with a Pseudomorphic InGaP Cap Layer," IEEE Photonics Technology Letters, Vol. 7, No. 8, pp. 914-916, August 1995.

8. R.N. Simons, Coplanar Waveguide Circuits, Components, and Systems, John Wiley \& Sons, Inc., New York, 2001.

9. J.B.D. Soole and H. Schumacher, "InGaAs Metal-Semiconductor-Metal Photodetectors for Long Wavelength Optical Communications," IEEE Journal of Quantum Electronics, Vol. 27, No. 3, pp. 737-751, March 1991.

10. B.C. Wadell, Transmission Line Design Handbook, p. 467, Artech House, Norwood, Massachusetts, 1991.

11. D.L. Rogers, "Integrated Optical Receivers using MSM Detectors," IEEE/OSA Journal of Lightwave Technology, Vol. 9, No. 12, pp. 1635-1638, December 1991.

12. R.N. Simons, Optical Control of Microwave Devices, Ch. 2 and Ch. 4, Artech House, Boston, 1990.

13. F. Riemenschneider, M. Maute, H. Halritter, G. Boehm, M.-C. Amann, and P. Meissner, "Continuously Tunable Long-Wavelength MEMS-VCSEL With Over 40-nm Tuning Range," IEEE Photonics Technology Letters, Vol. 16, No. 10, pp. 2212-2214, October 2004.

14. E. Hecht, Optics, p. 595, Addison Wesley, Boston, 2001.

15. S. Nakagawa, S.-Y. Hu, D.Louderback, and L.A. Coldren, "RF Crosstalk in Multiple-Wavelength Vertical-Cavity Surface-Emitting Laser Arrays," IEEE Photonics Technology Letters, Vol. 12, No. 6, pp. 612-614, June 2000.

16. X. Zheng, P.J. Marchand, D. Huang, O. Kibar, and S.C. Esener, "Cross Talk and Ghost Talk in a Microbeam Free-Space Optical Interconnect System with Vertical-Cavity Surface-Emitting Lasers, Microlenses, and Metal-Semiconductor-Metal Detectors," Applied Optics, Vol. 39, No. 26, pp. 48344841, September 2000. 


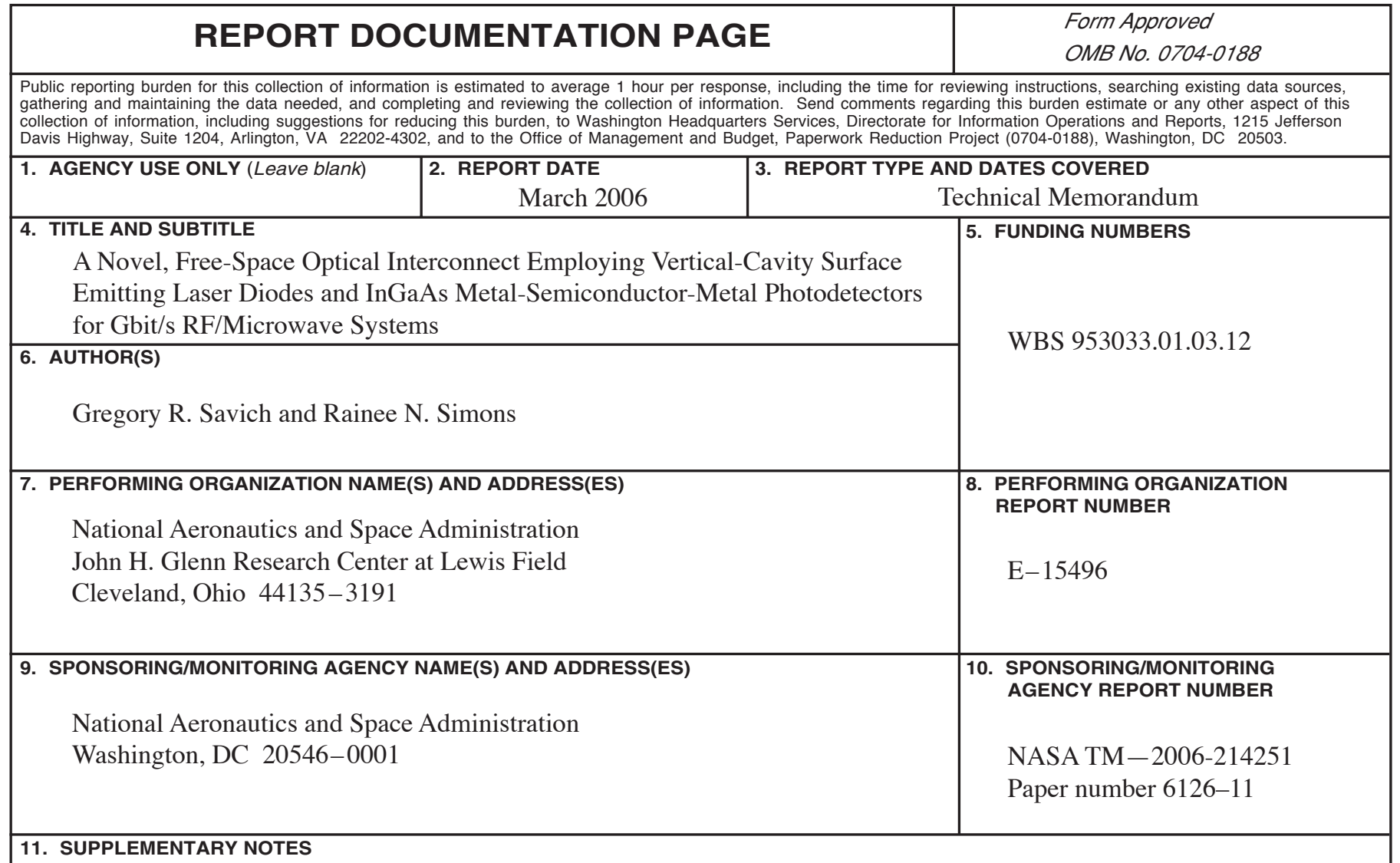

Prepared for Photonics West 2006, sponsored by the International Society for Optical Engineering, San Jose, California, January 21-26, 2006. Gregory R. Savich, University of Rochester, C.P.U. Box 276055, Rochester, New York, 14627; and Rainee N. Simons, NASA Glenn Research Center. Responsible person, Rainee N. Simons, organization code RCE, 216-433-3462.

12a. DISTRIBUTION/AVAILABILITY STATEMENT

12b. DISTRIBUTION CODE

Unclassified - Unlimited

Subject Category: 32

Available electronically at http://gltrs.grc.nasa.gov

This publication is available from the NASA Center for AeroSpace Information, 301-621-0390.

13. ABSTRACT (Maximum 200 words)

Emerging technologies and continuing progress in vertical-cavity surface emitting laser (VCSEL) diode and metalsemiconductor-metal (MSM) photodetector research are making way for novel, high-speed forms of optical data transfer in communication systems. VCSEL diodes operating at $1550 \mathrm{~nm}$ have only recently become commercially available, while MSM photodetectors are pushing the limits of contact lithography with interdigitated electrode widths reaching sub micron levels. We propose a novel, free-space optical interconnect operating at about 1Gbit/s utilizing VCSEL diodes and MSM photodetectors. We report on development, progress, and current work, which are as follows: first, analysis of the divergent behavior of VCSEL diodes for coupling to MSM photodetectors with a 50 by $50 \mu \mathrm{m}$ active area and second, the normalized frequency response of the VCSEL diode as a function of the modulating frequency. Third, the calculated response of MSM photodetectors with varying electrode width and spacing on the order of 1 to $3 \mu \mathrm{m}$ as well as the fabrication and characterization of these devices. The work presented here will lead to the formation and characterization of a fully integrated $1 \mathrm{Gbit} / \mathrm{s}$ free-space optical interconnect at $1550 \mathrm{~nm}$ and demonstrates both chip level and board level functionality for RF/microwave digital systems.

\begin{tabular}{|c|c|c|}
\hline \multicolumn{3}{|l|}{ 14. SUBJECT TERMS } \\
\hline $\begin{array}{l}\text { 17. SECURITY CLASSIFICATION } \\
\text { OF REPORT } \\
\text { Unclassified }\end{array}$ & $\begin{array}{l}\text { 18. SECURITY CLASSIFICATION } \\
\text { OF THIS PAGE } \\
\text { Unclassified }\end{array}$ & $\begin{array}{l}\text { 19. SECURITY CLASSIFICATION } \\
\text { OF ABSTRACT } \\
\text { Unclassified }\end{array}$ \\
\hline
\end{tabular}

NSN 7540-01-280-5500

Standard Form 298 (Rev. 2-89)

Prescribed by ANSI Std. Z39-18 298-102 

